\title{
Proteomic analysis to define predictors of treatment response to adalimumab or methotrexate in rheumatoid arthritis patients
}

\author{
Stephanie F. Ling $\mathbb{B}^{1,2} \cdot$ Nisha Nair $\mathbb{D}^{1} \cdot$ Suzanne M. M. Verstappen ${ }^{2,3} \cdot$ Anne Barton $\mathbb{B}^{1,2} \cdot$ Hans-Dieter Zucht ${ }^{4,5}$ • \\ Petra Budde ${ }^{4,5} \cdot$ Peter Schulz-Knappe ${ }^{4}$ MATURA consortium $\cdot$ Darren Plant ${ }^{1,2}$
}

Received: 26 July 2019 / Revised: 20 November 2019 / Accepted: 27 November 2019 / Published online: 10 December 2019

(c) The Author(s) 2019. This article is published with open access

\begin{abstract}
Seropositivity for anti-citrullinated peptide antibodies (ACPA) in patients with rheumatoid arthritis (RA), a chronic autoimmune arthritis, is associated with worse long-term disease outcomes. ACPA is ubiquitously tested in RA patients, but other autoantibodies exist (in both citrullinated and non-citrullinated form) which may provide additional information on RA subtypes and/or treatment response. We used a multiplex bead-based assay of 376 autoantibodies to test associations between these autoantibodies and treatment response in RA patients. Clusters of patients with similar autoantibody expression were defined and cluster membership was associated with treatment response. Thirty-four autoantibodies were differentially expressed in RA patients compared with healthy controls; citrullinated vimentin was associated with treatment response. A selection of citrullinated autoantibodies was found to be associated with treatment response in a subanalysis of ACPA-negative RA patients. Finer ACPA specificities in ACPA-negative RA patients may be predictive of treatment response and could represent a rich vein of future study.
\end{abstract}

\section{Introduction}

Rheumatoid arthritis (RA) is a chronic autoimmune arthritis that can also have multisystem involvement. RA pathogenesis involves numerous processes, including autoreactivity

For a full list of MATURA co-investigators, see Appendix.

Supplementary information The online version of this article (https:// doi.org/10.1038/s41397-019-0139-4) contains supplementary material, which is available to authorized users.

$\triangle$ Darren Plant

darren.plant@manchester.ac.uk

1 Centre for Genetics and Genomics Versus Arthritis, Centre for Musculoskeletal Research, The University of Manchester, Manchester, UK

2 NIHR Manchester Biomedical Research Centre, Manchester University NHS Foundation Trust, Manchester Academic Health Science Centre, Manchester, UK

3 Centre for Epidemiology Versus Arthritis, Centre for Musculoskeletal Research, The University of Manchester, Manchester, UK

4 Formerly, Protagen AG, Dortmund, Germany

5 Oncimmune Germany GmbH, Dortmund, Germany of T cells and autoantibody formation [1]. Testing of two of these autoantibodies, rheumatoid factor (RF) and anticitrullinated peptide antibodies (ACPA), aid both diagnosis and prognosis and form part of both European and British guidelines for management $[2,3]$. In the healthy population, ACPA was found to be positive in $0.8 \%$ of a population of 40,136 individuals in a large Dutch study [4], and RF has been estimated as positive in 5\% of healthy 50 -year-olds and 10-25\% of healthy 70-year-olds [5]. Seropositivity for ACPA is associated with worse long-term disease outcomes in RA [6], and it is important to achieve clinical remission in these patients as quickly as possible to prevent joint damage and disability. In addition, RF and ACPA seropositivity are associated with reduced response to anti-TNF drugs (a class of biological disease-modifying anti-rheumatic drug, bDMARD) [7].

Whilst RF and ACPA are known to be prognostic indicators in RA, those patients who are seronegative for RF/ ACPA are considered to be less at risk of a severe disease course. For example, in a primary care inception cohort of inflammatory arthritis patients recruited between 1990 and 1994, it was found that ACPA-positive patients had less benefit from treatment than ACPA-negative patients [8]. More recently, however, RF seronegativity was associated with nonresponse to methotrexate (a conventional synthetic 
DMARD, csDMARD) in a cohort of bDMARD-naïve patients [9].

Although ACPA and RF are the most investigated autoantibodies and are included in criteria for RA, other autoantibodies have been associated with RA prognosis, such as anti-carbamylated protein (anti-CarP) antibodies, which have been found to be associated with a more severe clinical course in RA patients who are seronegative for ACPA [10]. In addition, the search for predictors of prognosis and/or treatment response in RA patients remains an area of development, particularly in those who are RF/ ACPA seronegative. Most previous studies have used the commercial CCP2 assay to classify ACPA positivity, but other autoantibodies, both citrullinated and non-citrullinated exist, which may provide additional information on subtypes of RA and/or treatment response.

We used a multiplex bead-based approach to analyse sera from healthy controls (HC) and RA patients to determine: (1) similarities between RA patients in autoantibody expression and whether co-expression profiles are related to treatment outcomes; (2) differentially expressed autoantibodies between RA patients and $\mathrm{HC}$; and (3) whether any of the autoantibodies more frequently expressed in RA patients were associated with treatment response.

\section{Materials/Subjects and methods}

\section{Study subjects}

$\mathrm{HC}$ were chosen from specimens from blood donors of the Bavarian Red Cross, Germany [11]. RA patients were recruited from two cohorts. Patients with early RA commencing methotrexate were recruited from the Rheumatoid Arthritis Medication Study (RAMS; approving ethics committee: Central Manchester Research Ethics Committee (now NRES Committee North West - Greater Manchester Central; REC reference number: 08/H1008/25), a UK-based multi-centre prospective study [9]. Patients were aged 18 years or over with a physician diagnosis of either RA or undifferentiated polyarthritis and were commencing methotrexate for the first time, either as monotherapy or in combination with other csDMARDs or oral steroids. Patients with current or previous exposure to a bDMARD were ineligible for recruitment. Patients with established RA commencing adalimumab, an anti-TNF bDMARD, were recruited from the Biologics in RA Genetics and Genomics Study Syndicate (BRAGGSS; approving ethics committee: Central Manchester Research Ethics Committee (now NRES Committee North West - Greater Manchester Central; REC reference number: 04/Q1403/37), a large UKbased multi-centre prospective study [7]. Patients were Caucasian and aged 18 years or over, fulfilling the
American College of Rheumatology 1987 revised criteria for the classification of RA [12] and with Disease Activity Score in 28 joints using C-reactive protein (DAS28-CRP) $\geq$ 5.1 (indicative of active disease) [13] despite treatment with two previous csDMARDs. Informed consent was obtained from all subjects.

Serum samples were collected at baseline in both RA cohorts. Serum samples from HC and RA were processed and stored by Protagen AG, Dortmund, Germany for autoantibody profiling. RA serum samples were sent to the Centre for Musculoskeletal Research, The University of Manchester, Manchester, UK for processing, storage and analysis. ACPA was measured on RA samples using a commercially available ELISA (CCP2, Axis-Shield Diagnostics Ltd, Dundee, UK).

Demographic and clinical data were obtained at baseline. For the purpose of this study, DAS28-CRP measured at 3 months in BRAGGSS patients and at 6 months in RAMS patients was used. Therapeutic response was defined as good, moderate or poor according to the European League Against Rheumatism (EULAR) response criteria [14] at these timepoints.

\section{Measurement of autoantibodies}

Bead-based antigen arrays were used for the multiplex analysis of $\operatorname{IgG}$ autoantibody reactivity against 376 recombinant human protein antigens associated with autoimmune disease to detect autoantibodies. Overall, 39 of the 376 autoantigens were in citrullinated form. The full list of antigens is provided in the Supplementary Information. Detailed methodology for protein expression and multiplex autoantibody measurement has previously been described [15]. In brief, antigens were produced in E. coli, purified and covalently coupled to magnetic carboxylated colourcoded beads (MagPlex ${ }^{\mathrm{TM}}$ microspheres, Luminex Corporation, Austin, Texas). Antigen-coupled beads were combined, incubated with probands' sera and after washing procedures, incubated with a secondary PE-labelled antihuman IgG antibody. The beads were washed again, then analysed in a FlexMap3D instrument (Luminex Corporation). The median fluorescence intensity (MFI) values, reflecting semi-quantitative autoantibody levels, were obtained for each colour-coded antigen-coupled bead and each sample. This procedure was carried out once for each sample.

\section{Statistical analysis}

MFI values for $\mathrm{HC}$ and RA were normalised and $\log 2$ transformed. The 95th percentile for each autoantibody in $\mathrm{HC}$ was used to determine whether an RA sample was positive/negative for that autoantibody. Proteins with $<10 \%$ 
frequency in RA patients were subsequently excluded from analysis.

Correlation analysis of autoantibody data using the Pearson method was carried out in RA patients to define clusters of patients with similar autoantibody profiles (co-prevalence analysis) as described previously [16]. Logistic regression was used to determine seropositive autoantibodies associated with membership of each defined cluster vs. all other patients outside the cluster of interest; both seropositivity for autoantibodies and cluster membership were analysed as binary variables. The Benjamini-Hochberg adjustment was used to correct for multiple testing and autoantibodies with a significance level of $p<0.05$ were retained. Associations between cluster membership and treatment outcomes at 3/6 months were analysed using: (i) linear regression for improvement in DAS28, with a negative value indicating an increased score i.e. worsening disease activity; (ii) logistic regression for good vs. moderate/poor and poor vs. moderate/good EULAR response. All regression analyses were adjusted for age, gender, disease duration and baseline DAS28.

Further analysis was carried out in a subset of patients with available ACPA data according to CCP2 assays. Linear regression was used to determine autoantibodies differing in MFI between RA and HC; $p$-values were adjusted using the Benjamini-Hochberg correction. Significant autoantibodies (adjusted $p<0.05$ ) were tested for associations with treatment outcomes in RA patients only using: (i) linear regression for improvement in DAS28; (ii) logistic regression for good-/poor-vs-all EULAR response. Multivariate models consisting of measures of treatment response as the dependent variable and various autoantibody expression profiles of interest as independent variables were defined, and the Akaike information criterion (AIC) was used to compare goodness-of-fit between models. Again, all regression was adjusted for age, gender, disease duration and baseline DAS28.

All statistical analysis was carried out in $\mathrm{R}$ v3.4.2 (https://www.r-project.org/).

\section{Results}

\section{Patient characteristics}

A total of $52 \mathrm{HC}, 150$ BRAGGSS patients and 136 RAMS patients (286 RA patients in total) were included in the initial analysis; the characteristics of study participants are summarised in Table 1. ACPA status, as measured using the commercial CCP2 assay previously described, was available in 168 RA patients (37 BRAGGSS, 131 RAMS), and 90 of these 168 patients were ACPA positive (53.6\%). Controls were similar to cases in age and gender, with no significant differences between the groups. Patients in the BRAGGSS cohort had longer disease duration (median 7.6 years [IQR 2.6, 7.2], compared with median 0.8 years [IQR $0.4,1.4]$ in RAMS) and higher DAS28 score at baseline (mean 5.18 (SD 0.89), compared with mean 4.20 (SD1.16) in RAMS), as expected in patients commencing a bDMARD in the UK.

\section{Co-expression analysis}

Following the exclusion of autoantibodies with $<10 \%$ seropositivity in RA patients, 181 autoantibodies were retained for analysis. Four clear clusters of patients were identified from co-prevalence analysis (Fig. 1). Autoantibodies associated with membership of various clusters are detailed in Table 2. In Cluster 1 (no ACPA reactivity), 10 autoantibodies were significantly associated with cluster

Table 1 Demographic and clinical characteristics of patients at baseline and EULAR response after 3/6 months of treatment.

\begin{tabular}{|c|c|c|c|c|c|}
\hline Characteristic & $\mathrm{HC}(n=52)$ & All RA $(n=286)$ & $p$-value (HC vs RA) & BRAGGSS $(n=150)$ & RAMS $(n=136)$ \\
\hline Age (years), mean (SD) & $51.0(9.2)$ & $59.0(12.8)$ & 0.12 & $58.0(11.7)$ & $60.0(13.8)$ \\
\hline Female sex, $n(\%)$ & $39(75.0 \%)$ & $212(74.1 \%)$ & 1.00 & $111(74.0 \%)$ & $101(74.3)$ \\
\hline $\begin{array}{l}\text { Disease duration (years), } \\
\text { median [IQR] }\end{array}$ & - & $2.1[0.8,9.0]$ & - & $7.6[2.6,17.2]$ & $0.8[0.4,1.4]$ \\
\hline ACPA positive, $n(\%)$ & - & $\begin{array}{l}90(53.6)[118 \\
\text { missing] }\end{array}$ & - & $\begin{array}{l}24(64.9)[113 \\
\text { missing] }\end{array}$ & 66 (50.4) [5 missing] \\
\hline Baseline DAS28, mean (SD) & - & $4.71(1.14)$ & - & $5.18(0.89)$ & $4.20(1.16)$ \\
\hline EULAR response at 3 months & - & & - & & \\
\hline Good, $n(\%)$ & & $117(40.9)$ & & $58(38.7)$ & $59(43.4)$ \\
\hline Moderate, $n(\%)$ & & $60(21.0)$ & & $58(38.7)$ & $2(1.5)$ \\
\hline Poor, $n(\%)$ & & $109(38.1)$ & & $34(22.7)$ & $75(55.2)$ \\
\hline
\end{tabular}

BRAGGSS patients were treated with adalimumab for 3 months; RAMS patients were treated with methotrexate for 6 months and were bDMARD-naïve 


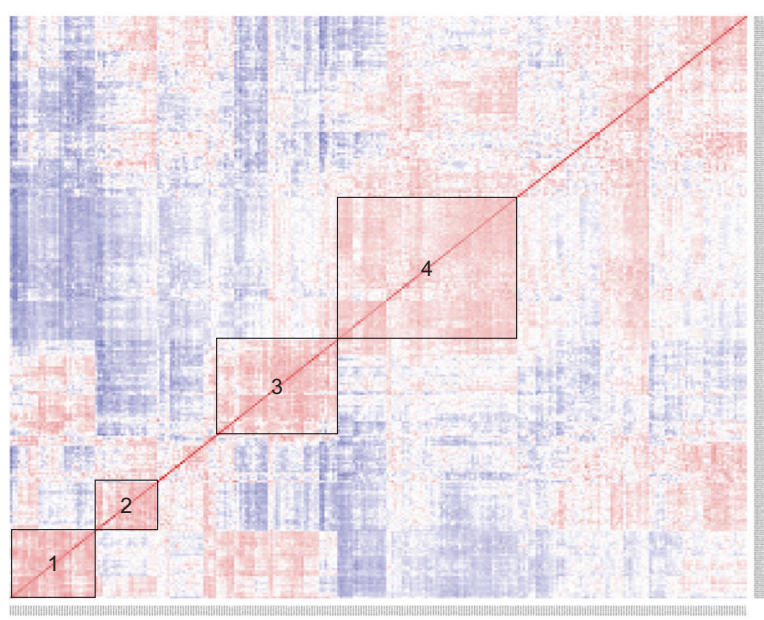

Fig. 1 Co-prevalence heatmap displaying four distinct clusters of RA patients with similar expression of autoantibodies. Red signatures demonstrate similar seropositivity of autoantibodies, whereas blue signatures represent patients with dissimilar seropositivity of autoantibodies.

membership; three of these were unique to Cluster 1 : cathepsin L1 (CTSL), Toll-like receptor 2 (TLR2) and interleukin (IL)-15. Cluster 2 membership was only associated with one autoantibody, complement C4-B (C4B). None of the autoantibodies associated with either Cluster 1 or Cluster 2 were in citrullinated form. Cluster 3 (moderate ACPA reactivity, cross-reactivity with Clusters 1 and 4) was associated with 11 autoantibodies, but these overlapped with those associated with either Cluster 1 (all non-citrullinated) or Cluster 4 (all citrullinated). Cluster 4 patients (high ACPA reactivity) were associated with 20 autoantibodies, 16 of which were unique to Cluster 4 (see Table 2) and all significantly associated autoantibodies were in citrullinated form. All citrullinated antibodies associated with Clusters 3 and 4 were differentially expressed in RA patients compared with controls, apart from citrullinated protein disulphide-isomerase A6 (PDIA6) in Cluster 4.

Results of analysis of cluster membership with change in DAS28 at 3/6 months are detailed in Table 3. Clusters 1 and 2 showed a non-significant trend towards increased DAS28 at $3 / 6$ months (i.e. worsening disease activity). Clusters 3 and 4 showed a non-significant trend towards DAS28 improvement at $3 / 6$ months. Due to proximity on the original co-expression heatmap, Clusters 1 and 2 were combined, as were Clusters 3 and 4. Clusters $1 / 2$ still showed a non-significant trend towards increased DAS28, but Clusters $3 / 4$ demonstrated a significant association with improved DAS28 at $3 / 6$ months (coefficient 0.38 , 95\% confidence intervals (CI) $0.08-0.69$, adjusted $R^{2} 0.3047, p=0.013$ ). Cluster membership was not associated with good or poor EULAR response, either separately or when Clusters $1 / 2$ and 3/4 were combined (Supplementary Table 1).
Table 2 Seropositive autoantibodies associated with patient membership in each cluster.

\begin{tabular}{|c|c|c|c|}
\hline Autoantibody & $\mathrm{OR}_{\mathrm{adj}}(95 \% \mathrm{CI})$ & $p$-value & Adj $p$-value \\
\hline \multicolumn{4}{|c|}{ Cluster $1(n=33)-$ no ACPA reactivity } \\
\hline IL6R & $36.33(13.13-100.55)$ & $4.59 \mathrm{E}-12$ & $<1 \mathrm{E}-06$ \\
\hline IL17RA & $23.39(9.53-57.39)$ & $5.80 \mathrm{E}-12$ & $<1 \mathrm{E}-06$ \\
\hline CD86 & $19.21(8.13-45.37)$ & $1.58 \mathrm{E}-11$ & $<1 \mathrm{E}-06$ \\
\hline CSF2RA & $20.08(8.38-48.10)$ & $1.71 \mathrm{E}-11$ & $<1 \mathrm{E}-06$ \\
\hline CD80 & $18.66(7.87-44.21)$ & $2.95 \mathrm{E}-11$ & $<1 \mathrm{E}-06$ \\
\hline CCL2 & $17.74(7.52-41.86)$ & $5.10 \mathrm{E}-11$ & $<1 \mathrm{E}-06$ \\
\hline TNFSF13 & $16.49(7.04-38.59)$ & $1.06 \mathrm{E}-10$ & $<1 \mathrm{E}-06$ \\
\hline CTSL $^{\mathrm{a}}$ & $3.44(1.58-7.50)$ & $1.90 \mathrm{E}-03$ & 0.023 \\
\hline $\mathrm{TLR}^{\mathrm{a}}$ & $3.56(1.51-8.38)$ & $3.72 \mathrm{E}-03$ & 0.037 \\
\hline $\operatorname{IL} 15^{\mathrm{a}}$ & $3.18(1.43-7.06)$ & $4.44 \mathrm{E}-03$ & 0.040 \\
\hline \multicolumn{4}{|c|}{ Cluster $2(n=24)-C 4 B$ reactivity } \\
\hline $\mathrm{C} 4 \mathrm{~B}^{\mathrm{a}}$ & $6.11(2.35-15.92)$ & $2.08 \mathrm{E}-04$ & 0.019 \\
\hline \multicolumn{4}{|c|}{$\begin{array}{l}\text { Cluster } 3(n=47) \text { - moderate ACPA reactivity, cross-reactivity with Clusters } 1 \\
\text { and } 4\end{array}$} \\
\hline CSF2RA & $9.50(4.44-20.34)$ & $6.66 \mathrm{E}-09$ & $1 \mathrm{E}-06$ \\
\hline CD80 & $9.27(4.34-19.79)$ & $8.86 \mathrm{E}-09$ & $1 \mathrm{E}-06$ \\
\hline IL17RA & $8.76(4.17-18.41)$ & $1.03 \mathrm{E}-08$ & $1 \mathrm{E}-06$ \\
\hline CCL2 & $8.96(4.20-19.11)$ & $1.41 \mathrm{E}-08$ & $1 \mathrm{E}-06$ \\
\hline TNFSF13 & $9.00(4.14-19.56)$ & $2.86 \mathrm{E}-08$ & $1 \mathrm{E}-06$ \\
\hline CD86 & $8.60(4.00-18.50)$ & $3.65 \mathrm{E}-08$ & $1 \mathrm{E}-06$ \\
\hline IL6R & $6.99(3.43-14.25)$ & $8.87 \mathrm{E}-0$ & $2 \mathrm{E}-06$ \\
\hline VIM_c & $3.78(1.89-7.59)$ & $1.78 \mathrm{E}-04$ & 0.004 \\
\hline SPP1_c & $3.23(1.64-6.36)$ & $6.91 \mathrm{E}-04$ & 0.014 \\
\hline EIF4H_c & $3.25(1.55-6.82)$ & $1.82 \mathrm{E}-03$ & 0.033 \\
\hline CLU_c & $2.78(1.45-5.36)$ & $2.20 \mathrm{E}-03$ & 0.036 \\
\hline \multicolumn{4}{|c|}{ Cluster $4(n=70)-$ high ACPA reactivity } \\
\hline SPP1_c & $20.48(9.30-45.10)$ & $6.37 \mathrm{E}-14$ & $<1 \mathrm{E}-06$ \\
\hline RBMS1_c $\mathrm{c}^{\mathrm{a}}$ & $13.92(6.97-27.80)$ & $8.89 \mathrm{E}-14$ & $<1 \mathrm{E}-06$ \\
\hline VIM_c & $30.17(12.25-74.27)$ & $1.25 \mathrm{E}-13$ & $<1 \mathrm{E}-06$ \\
\hline DNAJB1_c $c^{\mathrm{a}}$ & $12.83(6.47-25.42)$ & $2.60 \mathrm{E}-13$ & $<1 \mathrm{E}-06$ \\
\hline HNRNPA1_c ${ }^{\mathrm{a}}$ & $10.54(5.55-20.02)$ & $6.37 \mathrm{E}-13$ & $<1 \mathrm{E}-06$ \\
\hline FN1_c $c^{\mathrm{a}}$ & $10.23(5.43-19.30)$ & $6.58 \mathrm{E}-13$ & $<1 \mathrm{E}-06$ \\
\hline TRA2B_c ${ }^{\mathrm{a}}$ & $10.43(5.36-20.29)$ & $5.04 \mathrm{E}-12$ & $<1 \mathrm{E}-06$ \\
\hline CLU_c & $9.29(4.85-17.78)$ & $1.69 \mathrm{E}-11$ & $<1 \mathrm{E}-06$ \\
\hline NONO_c ${ }^{a}$ & $17.17(7.28-40.57)$ & $8.55 \mathrm{E}-11$ & $<1 \mathrm{E}-06$ \\
\hline SFPQ_c $c^{a}$ & $6.92(3.76-12.75)$ & $5.35 \mathrm{E}-10$ & $<1 \mathrm{E}-06$ \\
\hline CPSF6_c ${ }^{\mathrm{a}}$ & $6.92(3.76-12.75)$ & $1.74 \mathrm{E}-09$ & $<1 \mathrm{E}-06$ \\
\hline TNC_c $c^{a}$ & $5.99(3.23-11.11)$ & $1.36 \mathrm{E}-08$ & $<1 \mathrm{E}-06$ \\
\hline AEBP1_c ${ }^{\mathrm{a}}$ & $5.12(2.76-9.52)$ & $2.36 \mathrm{E}-07$ & $3 \mathrm{E}-06$ \\
\hline EIF4H_c & 130.97 (17.44-983.47) & $2.15 \mathrm{E}-06$ & $2.8 \mathrm{E}-05$ \\
\hline ASMTL_c ${ }^{a}$ & $7.45(10.59-581.40)$ & $1.96 \mathrm{E}-05$ & $2.37 \mathrm{E}-04$ \\
\hline PDIA6_c $\mathrm{c}^{\mathrm{a}}$ & $4.74(2.29-9.80)$ & $2.79 \mathrm{E}-05$ & $3.16 \mathrm{E}-04$ \\
\hline FGB_c $c^{a}$ & $3.49(1.92-6.34)$ & $4.34 \mathrm{E}-05$ & 4.62E-04 \\
\hline DDX5_c $c^{a}$ & $3.79(1.97-7.26)$ & $6.14 \mathrm{E}-05$ & $6.17 \mathrm{E}-04$ \\
\hline ACTB_c $c^{a}$ & $3.33(1.63-6.82)$ & $9.86 \mathrm{E}-04$ & 0.001 \\
\hline SRSF7_c ${ }^{a}$ & $2.94(1.52-5.70)$ & $1.35 \mathrm{E}-03$ & 0.012 \\
\hline
\end{tabular}

anique to cluster, "_c" suffix denotes autoantibody in citrullinated form.

\section{Expression of autoantibodies in patients with available ACPA status}

When compared with HC, 34 autoantibodies were differentially expressed in RA patients, only five of which were in 
Table 3 Associations between cluster membership and DAS28 improvement at 3/6 months, adjusted for age, gender, disease duration and baseline DAS28.

\begin{tabular}{lccc}
\hline Cluster & $\begin{array}{l}\text { Coefficient (95\% confidence } \\
\text { intervals) }\end{array}$ & Adj $R^{2}$ & $p$-value \\
\hline 1 & $-0.36(-0.83-0.11)$ & 0.2949 & 0.133 \\
2 & $-0.22(-0.77-0.33)$ & 0.2908 & 0.429 \\
3 & $0.27(-0.14-0.68)$ & 0.2934 & 0.200 \\
4 & $0.31(-0.04-0.66)$ & 0.2969 & 0.081 \\
Cluster 1/2 & $-0.34(-0.71-0.04)$ & 0.2969 & 0.080 \\
Cluster 3/4 & $0.38(0.08-0.69)$ & 0.3047 & 0.013 \\
\hline
\end{tabular}

a non-citrullinated form (Table 4). All autoantibodies were increased in RA, apart from one, tumour necrosis factor ligand superfamily member 13 (TNFSF13), which had reduced expression in RA patients when compared with HC.

All 34 autoantibodies were included in multivariate regression models to determine any associations with treatment outcomes at 3/6 months. Citrullinated heterogeneous nuclear ribonucleoprotein A1 (HNRNPA1) was significantly associated with DAS28 improvement (coefficient $0.69,95 \%$ CI $0.05-1.34, p=0.037$, see Supplementary Table 2). No other autoantibodies were associated with DAS28 improvement. ACPA alone (as assessed by positivity on a commercial CCP2 assay) was the best predictor of DAS28 improvement in a model adjusted for age, gender, disease duration and baseline DAS28 (coefficient 0.50, 95\% CI $0.11-0.91, p=0.014$; AIC 572.94 vs multivariate autoantibody model AIC 599.54).

In multivariate models of all 34 autoantibodies and good and poor EULAR response (see Supplementary Tables 3 and 4 , respectively), citrullinated vimentin was significantly associated with a poor EULAR response at 3/6 months, and also with reduced odds of achieving good EULAR response $\left(\mathrm{OR}_{\text {adj }} 4.19,95 \% \mathrm{CI} 1.07-18.32, p=0.046\right.$ and $\mathrm{OR}_{\text {adj }} 0.22$, $95 \%$ CI $0.05-0.81, p=0.030$, respectively) - see Fig. 2. A model using ACPA as measured using the CCP2 assay as the independent variable (without the 34 differentially expressed autoantibodies) was significantly associated with good EULAR response $\left(\mathrm{OR}_{\text {adj }} 2.40,95 \%\right.$ CI 1.24-4.77, $p=0.010$ ), with improved model fit compared with the multivariate autoantibody model (AIC 224.07, vs AIC 267.63 in multivariate autoantibody model). ACPA was also significantly associated with reduced odds of poor EULAR response $\left(\mathrm{OR}_{\text {adj }} 0.40,95 \%\right.$ CI $0.19-0.79, p=$ 0.010), again with improved model fit (AIC 208.04 vs AIC 249.27 in multivariate autoantibody model).

\section{Subanalysis in ACPA-negative RA patients}

Subanalysis was carried out in the 78 ACPA-negative RA patients. Citrullinated cleavage and polyadenylation specificity factor subunit 6 (CPSF6) was significantly associated with worse DAS28 at $3 / 6$ months (coefficient -1.83 , 95\% CI $(-3.60)-(-0.06), p=0.049$; Fig. 3$)$. In addition, citrullinated DnaJ homologue subfamily B member 1 (DNAJB1) was significantly associated with DAS28 improvement at 3/6 months (coefficient 2.17, 95\% CI $0.56-3.78, p=0.012$ ). None of the 34 differentially expressed autoantibodies between RA and HC were associated with EULAR response, neither good nor poor.

\section{Discussion}

This study used multivariate analytical techniques to interrogate a high-dimensional proteomic dataset linked to detailed clinical characteristics of RA patients. We identified distinct clusters of RA patients according to autoantibody profiles: these clusters do not appear to relate strongly to treatment response, but further exploration will be required to determine whether they are associated with other outcome measures that have not been collected in the current cohorts. We also found that testing individual autoantibodies adds nothing over the known correlation of a positive test using a commercial CCP2 assay in ACPApositive RA patients. However, in ACPA-negative patients, a trend was observed for the association between treatment response and seropositivity of citrullinated CPSF6 and citrullinated DNAJB1; this requires replication.

In the co-expression analysis, patients who were seronegative for citrullinated forms of autoantibodies were more likely to have a worse DAS28 after $3 / 6$ months of treatment. Conversely, patients with seropositivity for citrullinated forms of autoantibodies were more likely to have an improved DAS28. Citrullination is the result of a post-translational modification involving the conversion of the amino acid arginine into the amino acid citrulline. The citrullinated forms of autoantibodies recognised in this study are likely to represent finer specificities of ACPA beyond conventional commercially available assays, such as the CCP2 ELISA. We found that patients seropositive for finer ACPA specificities were more likely to demonstrate treatment response with either methotrexate or adalimumab. This could be because patients were treated more aggressively due to known ACPA seropositivity e.g. by more rapid escalation of methotrexate dosage or more timely escalation to bDMARD therapy. However, this has not been objectively confirmed as yet.

A total of 34 autoantibodies were found to be differentially expressed in RA patients vs. HC, and the majority of these (29/34) were in citrullinated form, which is unsurprising given the susceptibility of citrullinated proteins to an autoimmune response. Whilst two autoantibodies (citrullinated HNRNPA1 and citrullinated vimentin) were 
Table 4 Differentially expressed autoantibodies between RA patients and healthy controls, adjusted for age and gender.

\begin{tabular}{|c|c|c|c|c|}
\hline Protein & Coefficient & $95 \%$ confidence interval & p-value & Adjusted p-value (Benjamini-Hochberg correction) \\
\hline ASMTL_c & 3.41 & $2.50-4.33$ & $4.66 \mathrm{E}-12$ & $<1 \mathrm{E}-07$ \\
\hline EIF4H_c & 2.72 & $1.94-3.51$ & $9.09 \mathrm{E}-11$ & $<1 \mathrm{E}-07$ \\
\hline SPP1_c & 2.05 & $1.43-2.67$ & $5.53 \mathrm{E}-10$ & $<1 \mathrm{E}-07$ \\
\hline NONO_c & 2.11 & $1.45-2.78$ & $2.43 \mathrm{E}-09$ & $<1 \mathrm{E}-07$ \\
\hline CLU_c & 1.87 & $1.25-2.49$ & $1.42 \mathrm{E}-08$ & $1 \mathrm{E}-06$ \\
\hline VIM_c & 2.52 & $1.67-3.37$ & $2.46 \mathrm{E}-08$ & $1 \mathrm{E}-06$ \\
\hline FN1_c & 1.71 & $1.11-2.31$ & $6.93 \mathrm{E}-08$ & $2 \mathrm{E}-06$ \\
\hline CPSF6_c & 2.17 & $1.41-2.93$ & $7.55 \mathrm{E}-08$ & $2 \mathrm{E}-06$ \\
\hline TRA2B_c & 1.39 & $0.86-1.93$ & $7.54 \mathrm{E}-07$ & $1.50 \mathrm{E}-05$ \\
\hline RBMS1_c & 1.61 & $0.99-2.24$ & $9.58 \mathrm{E}-07$ & $1.70 \mathrm{E}-05$ \\
\hline ACTB_c & 1.01 & $0.61-1.41$ & $1.93 \mathrm{E}-06$ & $3.20 \mathrm{E}-05$ \\
\hline HNRNPA1_c & 1.45 & $0.85-2.06$ & $4.42 \mathrm{E}-06$ & $6.70 \mathrm{E}-05$ \\
\hline DNAJB1_c & 1.38 & $0.76-2.01$ & $2.24 \mathrm{E}-05$ & $3.12 \mathrm{E}-04$ \\
\hline TNC_c & 1.18 & $0.62-1.74$ & $4.92 \mathrm{E}-05$ & $6.37 \mathrm{E}-04$ \\
\hline FGB_c & 0.92 & $0.45-1.39$ & $1.64 \mathrm{E}-04$ & $1.98 \mathrm{E}-03$ \\
\hline SFPQ_c & 1.12 & $0.52-1.72$ & $3.03 \mathrm{E}-04$ & $3.43 \mathrm{E}-03$ \\
\hline SRSF7_c & 0.77 & $0.35-1.19$ & $3.57 \mathrm{E}-04$ & $3.68 \mathrm{E}-03$ \\
\hline TUBB_c & 0.72 & $0.33-1.12$ & $3.66 \mathrm{E}-04$ & $3.68 \mathrm{E}-03$ \\
\hline PADI4_c & 0.69 & $0.31-1.06$ & $4.50 \mathrm{E}-04$ & $4.28 \mathrm{E}-03$ \\
\hline AEBP1_c & 0.91 & $0.36-1.46$ & $1.39 \mathrm{E}-03$ & $1.26 \mathrm{E}-02$ \\
\hline DDX5_c & 0.81 & $0.31-1.31$ & $1.66 \mathrm{E}-03$ & $1.43 \mathrm{E}-02$ \\
\hline TNFSF13 & -1.17 & $-1.94-(-0.39)$ & $2.62 \mathrm{E}-03$ & $2.98 \mathrm{E}-02$ \\
\hline APOE_c & 0.86 & $0.27-1.44$ & $4.31 \mathrm{E}-03$ & $3.26 \mathrm{E}-02$ \\
\hline RBM39_c & 0.61 & $0.20-1.02$ & $4.32 \mathrm{E}-03$ & $3.26 \mathrm{E}-02$ \\
\hline IL1B & 0.53 & $0.17-0.89$ & $4.53 \mathrm{E}-03$ & $3.27 \mathrm{E}-02$ \\
\hline TUBB & 0.56 & $0.17-0.94$ & $4.69 \mathrm{E}-03$ & $3.27 \mathrm{E}-02$ \\
\hline FGA_c & 0.53 & $0.16-0.91$ & $5.73 \mathrm{E}-03$ & $3.84 \mathrm{E}-02$ \\
\hline IGF1_c & 0.60 & $0.18-1.03$ & $6.11 \mathrm{E}-03$ & $3.91 \mathrm{E}-02$ \\
\hline FEN1_c & 0.66 & $0.19-1.12$ & $6.40 \mathrm{E}-03$ & $3.91 \mathrm{E}-02$ \\
\hline ENO1_c & 0.87 & $0.25-1.48$ & $6.48 \mathrm{E}-03$ & $3.91 \mathrm{E}-02$ \\
\hline MMP2 & 0.48 & $0.13-0.82$ & $7.05 \mathrm{E}-03$ & 4.12E-02 \\
\hline OBSL1 & 0.49 & $0.13-0.84$ & $7.73 \mathrm{E}-03$ & $4.37 \mathrm{E}-02$ \\
\hline HIST1H4A_c & 0.48 & $0.13-0.83$ & $8.32 \mathrm{E}-03$ & $4.56 \mathrm{E}-02$ \\
\hline PTBP1_c & 0.67 & $0.17-1.17$ & $9.07 \mathrm{E}-03$ & $4.83 \mathrm{E}-02$ \\
\hline
\end{tabular}

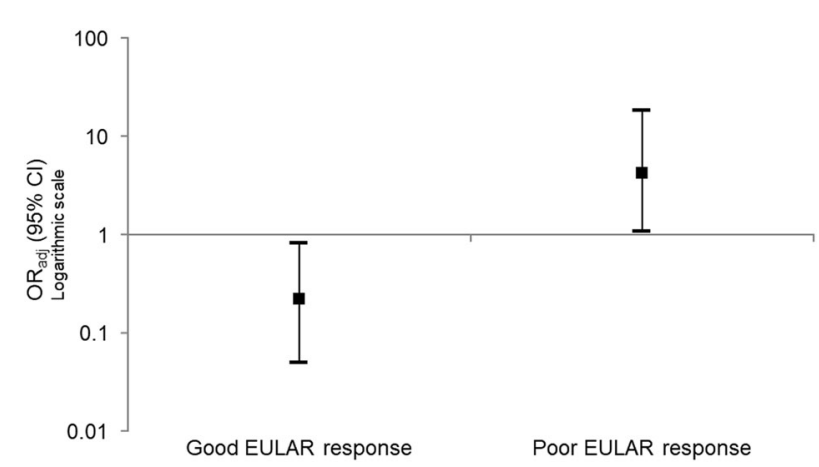

Fig. 2 Citrullinated vimentin is associated with good and poor EULAR response at 3/6 months in RA patients with available ACPA status. associated with DAS28 improvement after $3 / 6$ months of treatment, seropositivity for ACPA using the commercially available CCP2 assay remained the best predictor of treatment response, so the utility of these novel biomarkers is yet to be demonstrated over established practice.

Interestingly, a proportion of ACPA-negative patients were seropositive for citrullinated autoantibodies, and this may be an important area of future development in defining predictors of treatment response in RA. The presence of autoantibodies to citrullinated CPSF6 in ACPA-negative RA patients was associated with worsening DAS28 at 3/ 6 months. CPSF6 is a component of the cleavage factor IM (CFIm) complex that is involved in the maturation of 


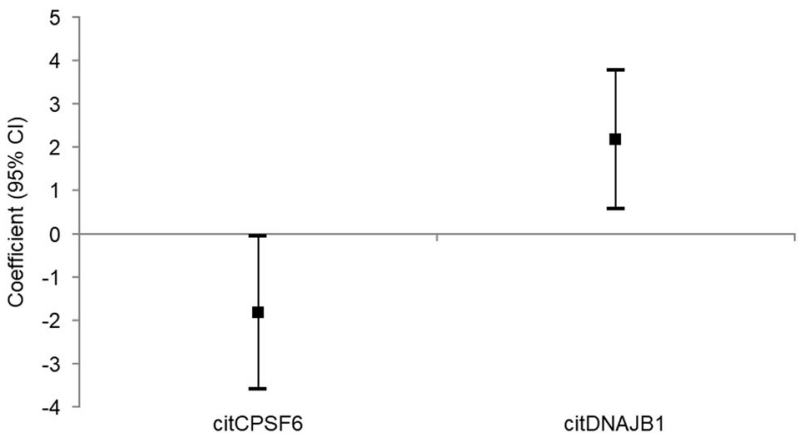

Fig. 3 Citrullinated CPSF6 is associated with worse DAS28 at 3/ 6 months and citrullinated DNAJB1 is associated with improved DAS28 at $3 / 6$ months in subanalysis of 78 ACPA-negative RA patients.

pre-mRNA into functional mRNA [17]. Autoantibodies to citrullinated DNAJB1 were associated with improved DAS28 at 3/6 months. DNAJB1 interacts with heat shock protein (HSP)70 and it is involved in the heat shock response [18]. Whilst these citrullinated antibodies are associated with treatment response in this cohort of RA patients, we cannot say for certain whether this relates to disease severity or is drug-specific.

Strengths of this study include recruitment of HC, meaning that analysis could be focused on autoantibodies differentially expressed by RA patients, enhancing interpretation. Furthermore, the RA patients recruited were from a well-phenotyped cohort with detailed clinical information. The RA patients were followed up over time and, therefore, longitudinal clinical information (i.e. response to medication over time) was included in analysis. However, results from the two cohorts, BRAGGSS and RAMS, were not presented separately due to sample size limitations.

In conclusion, finer ACPA specificities in ACPAnegative RA patients may be predictive of treatment response and could represent a rich vein of future study.

Acknowledgements This research was co-funded by the NIHR Manchester Biomedical Research Centre and MATURA (jointly funded by the Medical Research Council (grant code MR/K015346/1) and Versus Arthritis (grant code 20670). AB is an NIHR Senior Investigator. The views expressed are those of the authors and not necessarily those of the NHS, the NIHR or the Department of Health. The funders had no role in the study design, data collection and analysis, data interpretation, the writing of the manuscript or the decision to submit the manuscript for publication.

\section{Compliance with ethical standards}

Conflict of interest PB and HDZ are employees of Oncimmune Germany $\mathrm{GmbH}$, formerly Protagen AG. PSK is a previous employee and a shareholder of Protagen AG.

Publisher's note Springer Nature remains neutral with regard to jurisdictional claims in published maps and institutional affiliations.
Open Access This article is licensed under a Creative Commons Attribution 4.0 International License, which permits use, sharing, adaptation, distribution and reproduction in any medium or format, as long as you give appropriate credit to the original author(s) and the source, provide a link to the Creative Commons license, and indicate if changes were made. The images or other third party material in this article are included in the article's Creative Commons license, unless indicated otherwise in a credit line to the material. If material is not included in the article's Creative Commons license and your intended use is not permitted by statutory regulation or exceeds the permitted use, you will need to obtain permission directly from the copyright holder. To view a copy of this license, visit http://creativecommons. org/licenses/by/4.0/.

\section{References}

1. McInnes IB, Schett G. The pathogenesis of rheumatoid arthritis. N. Engl J Med. 2011;365:2205-19.

2. Combe B, Landewe R, Daien CI, Hua C, Aletaha D, Alvaro-Gracia JM, et al. 2016 update of the EULAR recommendations for the management of early arthritis. Ann Rheum Dis. 2017;76:948-59.

3. NICE. Rheumatoid arthritis in adults: management. 2018. https://www.nice.org.uk/guidance/ng 100/chapter/Recommenda tions.

4. van Zanten A, Arends S, Roozendaal C, Limburg PC, Maas F, Trouw LA, et al. Presence of anticitrullinated protein antibodies in a large population-based cohort from the Netherlands. Ann Rheum Dis. 2017;76:1184-90.

5. Ingegnoli F, Castelli R, Gualtierotti R. Rheumatoid factors: clinical applications. Dis Markers. 2013;35:727-34.

6. Forslind K, Ahlmen M, Eberhardt K, Hafstrom I, Svensson B, Group BS. Prediction of radiological outcome in early rheumatoid arthritis in clinical practice: role of antibodies to citrullinated peptides (anti-CCP). Ann Rheum Dis. 2004;63:1090-5.

7. Potter C, Hyrich KL, Tracey A, Lunt M, Plant D, Symmons DP, et al. Association of rheumatoid factor and anti-cyclic citrullinated peptide positivity, but not carriage of shared epitope or PTPN22 susceptibility variants, with anti-tumour necrosis factor response in rheumatoid arthritis. Ann Rheum Dis. 2009;68:69-74.

8. Farragher TM, Lunt M, Plant D, Bunn DK, Barton A, Symmons DP. Benefit of early treatment in inflammatory polyarthritis patients with anti-cyclic citrullinated peptide antibodies versus those without antibodies. Arthritis Care Res (Hoboken). 2010;62:664-75.

9. Sergeant JC, Hyrich KL, Anderson J, Kopec-Harding K, Hope $\mathrm{HF}$, Symmons DPM, et al. Prediction of primary non-response to methotrexate therapy using demographic, clinical and psychosocial variables: results from the UK Rheumatoid Arthritis Medication Study (RAMS). Arthritis Res Ther. 2018;20:147.

10. Shi J, Knevel R, Suwannalai P, van der Linden MP, Janssen GM, van Veelen PA, et al. Autoantibodies recognizing carbamylated proteins are present in sera of patients with rheumatoid arthritis and predict joint damage. Proc Natl Acad Sci USA. 2011;108:17372-7.

11. Zoglmeier C, Martin S, Weinauer F. The Bavarian Red Cross Blood Donor BioBank: the first successful combination of blood donation and biobanking for medical research. Transfusion. 2011;51:1121-2.

12. Arnett FC, Edworthy SM, Bloch DA, McShane DJ, Fries JF, Cooper NS, et al. The American Rheumatism Association 1987 revised criteria for the classification of rheumatoid arthritis. Arthritis Rheum. 1988;31:315-24.

13. Prevoo ML, van 't Hof MA, Kuper HH, van Leeuwen MA, van de Putte LB, van Riel PL. Modified disease activity scores that include twenty-eight-joint counts. Development and validation in 
a prospective longitudinal study of patients with rheumatoid arthritis. Arthritis Rheum. 1995;38:44-8.

14. van Gestel AM, Prevoo ML, van 't Hof MA, van Rijswijk MH, van de Putte LB, van Riel PL. Development and validation of the European League Against Rheumatism response criteria for rheumatoid arthritis. Comparison with the preliminary American College of Rheumatology and the World Health Organization/ International League Against Rheumatism Criteria. Arthritis Rheum. 1996;39:34-40.

15. Vordenbaumen S, Lueking A, Budde P, Zucht HD, Goehler H, Brinks R, et al. Sequential high-content profiling of the IgG- autoantibody repertoire reveals novel antigens in rheumatoid arthritis. Arthritis Res Ther. 2016;18:235.

16. Budde P, Zucht HD, Vordenbaumen S, Goehler H, Fischer-Betz $\mathrm{R}$, Gamer M, et al. Multiparametric detection of autoantibodies in systemic lupus erythematosus. Lupus. 2016;25:812-22.

17. Zhu Y, Wang X, Forouzmand E, Jeong J, Qiao F, Sowd GA, et al. Molecular mechanisms for CFIm-mediated regulation of mRNA alternative polyadenylation. Mol Cell. 2018;69:62-74 e4.

18. Shi Y, Mosser DD, Morimoto RI. Molecular chaperones as HSF1-specific transcriptional repressors. Genes Dev. 1998; 12:654-66. 